\title{
A Chandra Look at the Old Open Cluster M67
}

\author{
Maureen van den Berg, Gianpiero Tagliafferi, Tomaso Belloni \\ Osservatorio Astronomico di Brera, Via E. Bianchi 46, 23807 Merate \\ $(L C)$, Italy
}

\author{
Frank Verbunt \\ Astronomical Institute, Utrecht University, Postbox 80000, 3508 TA \\ Utrecht, The Netherlands
}

\begin{abstract}
We present the results of a Chandra observation of the old open cluster M $67 ; 25$ cluster members and 13 suspected members were detected, including 22 binaries. We believe that the X-rays of most systems can be explained by enhanced activity due to rapid rotation in a tidally locked orbit. This cannot be the case for the blue and yellow stragglers with orbital periods between 40 and $5000 \mathrm{~d}$, like the new source S 997 . We do not understand the X-rays of these long-period binaries.
\end{abstract}

\section{Introduction}

The X-rays of late-type stars are generated by a magnetic dynamo driven by convection and rotation. As single stars spin down as they age, their X-ray emission decreases accordingly. Old late-type stars in a binary can however rotate rapidly if the rotation is tidally locked to the orbit. ROSAT observations of old open clusters have indeed detected many active binaries among the cluster members. Therefore, observing old clusters in X-rays is a way to study homogeneous samples of active binaries, but also the properties of the clusters' binary populations, which in its turn is important to understand the dynamical evolution of clusters. Here we present the results of a 47-ks Chandra observation of the 4-Gyr-old open cluster M 67 with ACIS. M67 has been observed twice before in X-rays with ROSAT (see Belloni, Verbunt, \& Mathieu 1998).

\section{Results}

Chandra detected 158 sources, including 25 proper-motion cluster members (15 were already detected by ROSAT) and 13 sources without proper-motion information, that we suspect to be members from their position close to the M67 main sequence. All sources with optical counterparts are shown in Fig. 1a.

As expected, most (21) of the detected members are binaries while 4 have not been investigated for binarity; 2 sources on the lower main sequence are photometric variables with periods $<9 \mathrm{~d}$, which makes them good candidate active binaries. Fig. 1b shows the X-ray luminosity $L_{\mathrm{X}}$ of all M 67 binaries in the field of view versus orbital period $P_{\mathrm{b}}$. Most binaries with $P_{\mathrm{b}}<12 \mathrm{~d}$ were 

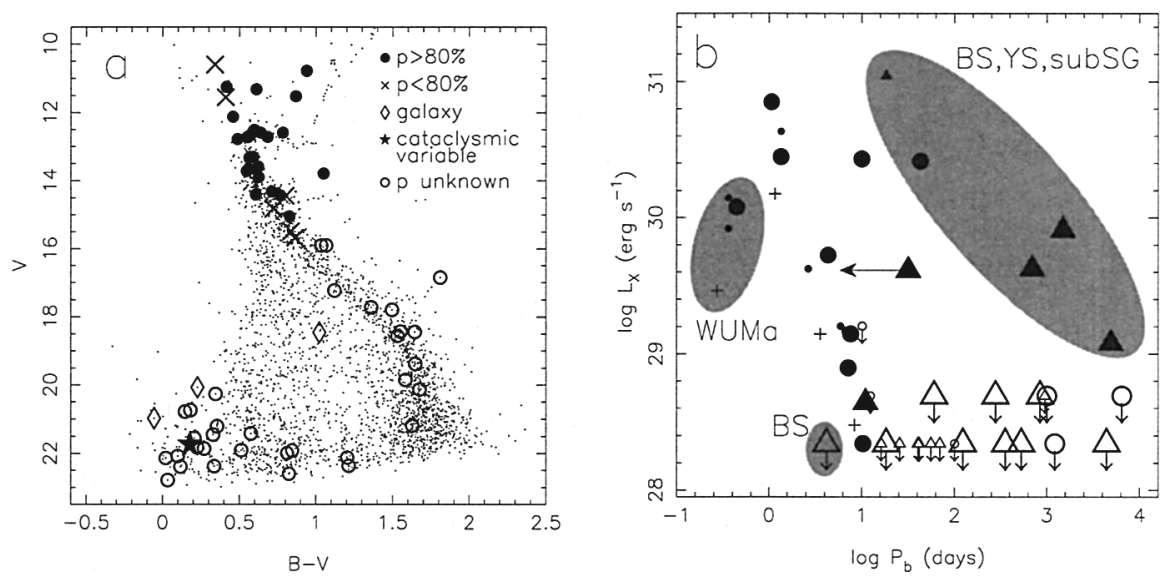

Figure 1. a) Colour-magnitude diagram of M67 with optical counterparts of Chandra X-ray sources; $p$ is the probability for cluster membership b) Logarithm of $L_{\mathrm{X}}(0.3-7 \mathrm{keV})$ versus $P_{\mathrm{b}}$ (see e.g. Mathieu et al. 1990) of the binaries in the field of view. Filled (open) symbols are detections (upper limits). Large symbols are for systems with $V<13$. Plus-signs are suspected members and/or binaries. Eccentric (circular) binaries are plotted with triangles (circles). $\mathrm{BS} / \mathrm{YS}=$ blue/yellow straggler, $\mathrm{SG}=$ subgiant, WUMa $=$ contact binary.

detected; this period corresponds to the period where the transition from circular to eccentric orbits is observed in M 67. In general, tidal synchronization is achieved before circularizaton (Zahn 1977), therefore these binaries are probably tidally locked and $P_{\mathrm{b}}$ is a good measure for the rotation period. $L_{\mathrm{X}}$ seems to decrease with increasing orbital period, although $\mathrm{S} 999$ is $\sim 100$ times more luminous than S 986 with a similar $P_{\mathrm{b}}(\sim 10 \mathrm{~d})$ and photometric properties. The 4 binaries with the shortest orbital periods are contact binaries, known to be less luminous in X-rays than single fast rotators (Stẹpien, Schmitt, \& Voges 2001). We detect 6 binaries with $P_{\mathrm{b}}>12 \mathrm{~d}$. S 1242 is eccentric, and the rotation could be synchronized to the orbital period near periastron (5.9 d; see arrow in Fig. 1b). The remaining 5 binaries are blue stragglers, yellow stragglers and a subsubgiant whose evolutionary histories may be complex. We do not understand the X-rays of these systems. Chandra detected one new long-period blue straggler: S 997 with an eccentric orbit of $4913 \mathrm{~d}$. Like the blue straggler S 1082, it may contain a close binary that is responsible for the X-rays (van den Berg et al. 2001).

\section{References}

Belloni, T., Verbunt, F., Mathieu, R.D. 1998, A\&A 339, 431

Stȩpień, K., Schmitt, J., Voges, W. 2001, A\&A 370, 157

van den Berg, M., Orosz, J., Verbunt, F., Stassun, K. 2001, A\&A 375, 375

Zahn, J.-P. 1977, A\&A 57, 383

Mathieu, R.D., Latham, D., Griffin, R. 1990, AJ 100, 1859 